\title{
FEATURES OF STIMULATED EMISSION OF A MEROCYANINE DYE IN THE PORES OF ANODIZED ALUMINUM
}

\author{
Ibrayev N.Kh. ${ }^{1}$, Afanasyev D.A. ${ }^{1,2}$, Omarova G.S. ${ }^{1}$ \\ ${ }^{1}$ Institute of Molecular Nanophotonics, E.A. Buketov Karaganda University, Karaganda, Kazakhstan, \\ guldenserikovna@mail.ru \\ 2Institute of Applied Mathematics, Karaganda, Kazakhstan
}

\begin{abstract}
The results of the study of the spectral-luminescent properties and the generation of stimulated emission of merocyanine dye molecules in a porous aluminum oxide films are presented. The addition of silver nanoparticles to the porous aluminum oxide films leads to an increase in the absorption cross section and an increase in the quantum yield of dye fluorescence in the alumina films. However, in the alumina films with silver nanoparticles, the generation of stimulated emission of dye molecules was not detected. We also studied the effect of polymethyl methacrylate polymer deposited on the surface of the alumina films with a dye on the spectral-luminescent properties and generation of stimulated emission of this dye. An increase in the optical density in the absorption band of the dye and an increase in the fluorescence intensity in samples with a deposited polymethyl methacrylate film are observed. The generation threshold of polymethine dye is reduced by a factor of 40 in the porous aluminum oxide sample with polymethyl methacrylate.
\end{abstract}

Keywords: porous aluminum oxide, electrochemical anodizing, merocyanine dye, stimulated emission, polymethyl methacrylate.

\section{Introduction}

Recently, the creation of active media based on nanostructured solid-state matrices doped with dye molecules has been a great attention. In this aspect, porous aluminum oxide (PAO) is one of the promising materials on the basis of which laser active media can be created. The films of PAO have a high specific surface area and thermal conductivity, and they are also optically transparent materials. The structure of the films is a system of tightly packed ordered pores. The pores are located perpendicular to the film surface. Diameter and distance between pores can be varied by changing the anodizing conditions [1]. If the cavities of cylindrical resonators are filled with organic dye molecules with a high fluorescence quantum yield, then such a system can be used as an active medium for a tunable laser. Stimulated emission has been obtained in porous aluminum oxide films for a number of dyes. It is shown that the use of metal nanoparticles (NPs) leads to a decrease in the generation threshold and an increase in the intensity of stimulated emission [2-4].

The aim of this work was to obtain metal-enhanced generation of a meracyanine dye in porous alumina films. For this, the PAO films were doped with silver and gold nanoparticles. However, the addition of silver and gold NPs didn't lead to the generation of stimulated emission of the samples. We studied the effect of a polymer film of polymethyl methacryolate (PMMA) applied to the surface of PAO with dye molecules. This promoted an increase in the stimulated emission intensity and a decrease in the generation threshold for dye molecules in PAO films with supported PMMA as compared to PAO without PMMA.

\section{Experimental part}

For the preparation of porous aluminum oxide, polished aluminum plates with a degree of purity (99.99\%), deionized water (purified using an AquaMax water purification system), ethanol (anhydrous) were used. The $\mathrm{H}_{2} \mathrm{SO}_{4}$ electrolyte was used for anodizing. All reagents were analytically pure and were used without further purification. The method of preparing aluminum plates and the anodizing process are detailed in the work [5].

Films of porous alumina were synthesized under conditions including two stages of anodization at a voltage of $\mathrm{U}=40 \mathrm{~V}$. The synthesis of $\mathrm{PAO}$ films was carried out in accordance with the procedure given in the works [3, 6]. Merocyanine dye 1 was used for the investigated (Fig.1). 
<smiles>CN1/C(=C/C=C/C=C/C=C(C#N)C#N)C(C)(C)c2ccccc21</smiles>

Fig.1. Merocyanine dye 1

The method for preparation and purification of the dye $\mathbf{1}$ is described in the work [7]. The choice of this dye is due a high photochemical stability, soluble in the solvents used in the work. Dye 1 also has stimulated emission in solutions. The surface morphology of the obtained PAO films was investigated on a Tescan Mira-3 (Tescan) scanning electron microscope (SEM). The absorption spectra of the solutions and films under study were measured on a Cary-300 spectrophotometer (Agilent). Fluorescence spectra were measured on a Cary Eclipse spectrofluorometer (Agilent). The lifetimes of the excited state of the dye molecules were measured using a time-correlated photon counting pulsed spectrofluorimeter (Becker \& Hikl, Germany). The fluorescence quantum yield $\left(\varphi_{\mathrm{f}}\right)$ of the dye was measured by the absolute method using an integrating sphere (Avantes).

\section{Discussion of results}

Optical properties of PAO films doped with silver NPs and molecules of the dye $\mathbf{1}$ were studied. The properties of stimulated emission of meracyanine $\mathbf{1}$ in PAO and the effect of silver NPs on this properties are studied. The concentration of Ag nanoparticles in PAO films was varied concentration of the precursor of silver NPs (silver nitrate). Precursor concentrations of $0.002 \mathrm{M}$ and $0.02 \mathrm{M}$ were used. The corresponding films were designated conventionally I and II. The pore diameter is on average 15-20 $\mathrm{nm}$ (Fig. 2, a).

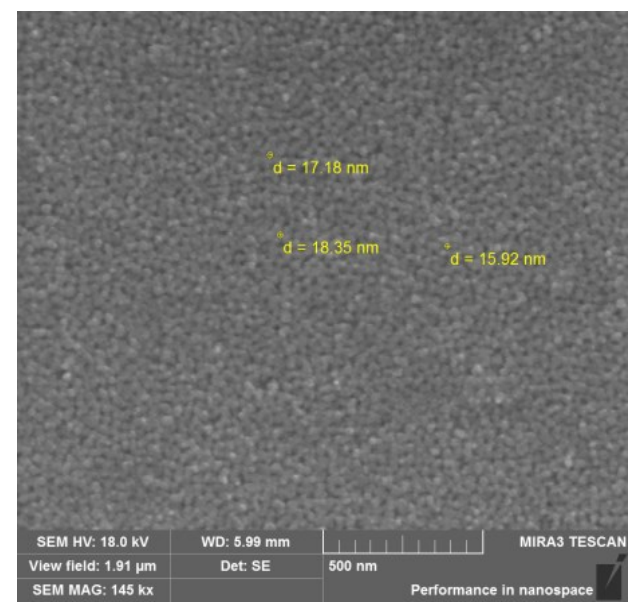

a

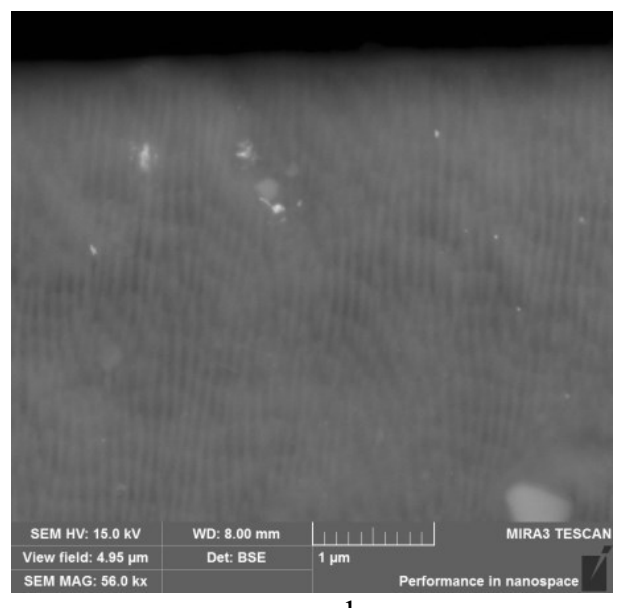

b

Fig.2. SEM images of the surface of the PAO film (a) and a transverse chipping (b) of the sample obtained by anodizing in sulfuric acid at $15 \mathrm{~V}$.

Images of PAO films doped with silver NPs are shown in Fig. 2. Light particles on the surface and in pores are silver NPs, which is confirmed by the results of X-ray spectral microanalysis (Fig. 3, c). The absorption spectra of the dye in the PAO films are shown in Fig. 4. The maximum fluorescence of the dye is at $652 \mathrm{~nm}$. The fluorescence intensity depends on the concentration of NPs in the pores. The maximum luminescence intensity is observed in films I (Fig. 4). For dye 1 in solution and in films, the fluorescence quantum yield $\left(\varphi_{\mathrm{f}}\right)$ was determined. In ethanol solution, the quantum yield of the dye was $\varphi_{\mathrm{f}}=0.05$ at $\mathrm{C}_{\mathrm{cr}}=10^{-6} \mathrm{~mol} / \mathrm{L}$ and $\varphi_{\mathrm{f}}=0.01$ at $\mathrm{C}_{\mathrm{cr}}=10^{-4} \mathrm{~mol} / \mathrm{L}$. These values of the quantum yield are close to the values obtained in the work [8]. The fluorescence quantum yield of the dye in PAO films has similar values. For example, in PAO films without silver NPs $\varphi_{\mathrm{f}}=0.003$. In the presence of silver NPs, the luminescence quantum yield increases to $\varphi_{\mathrm{f}}=0.015$ for the films I and $\varphi_{\mathrm{f}}=0.004$ for the films II. The decay of the luminescence of the dye in the solution occurs exponentially with $\tau_{\mathrm{f}}=0.4$ ns (Fig. 5 , a). 


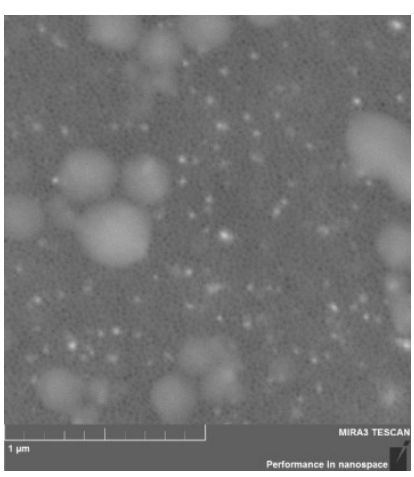

a

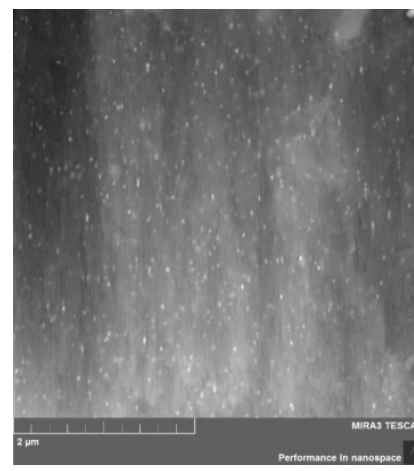

b

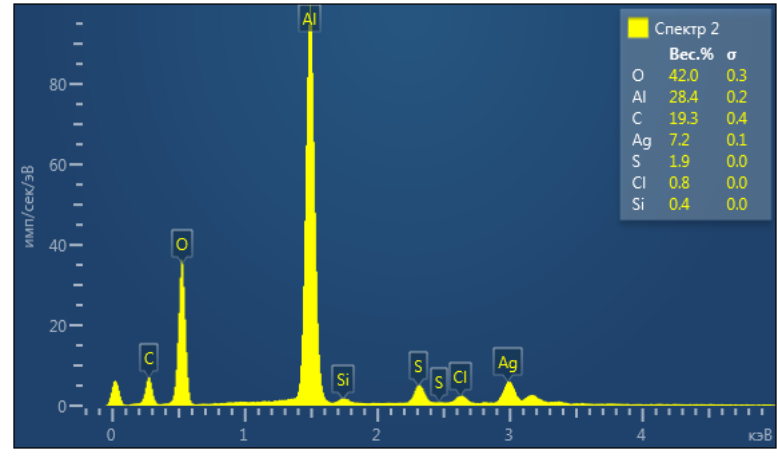

c

Fig.2. SEM images of the surface (a), chipping (b), and X-ray spectrum (c) of the PAO film doped with silver NPs.

$\mathrm{D}$

I, a.u.

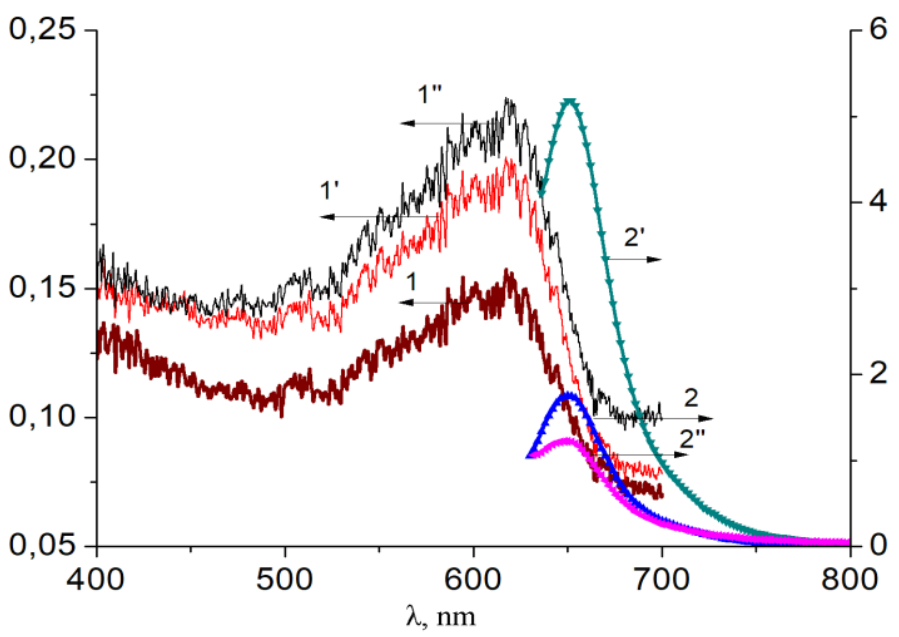

Fig.4. Absorption spectrum (1, 1', 1' ') and fluorescence (2, 2', 2 ") of the dye in POA films:

1,2 - spectra of the dye in PAO without silver NPs; 1', 2' - sample I; 1", 2 " - sample II

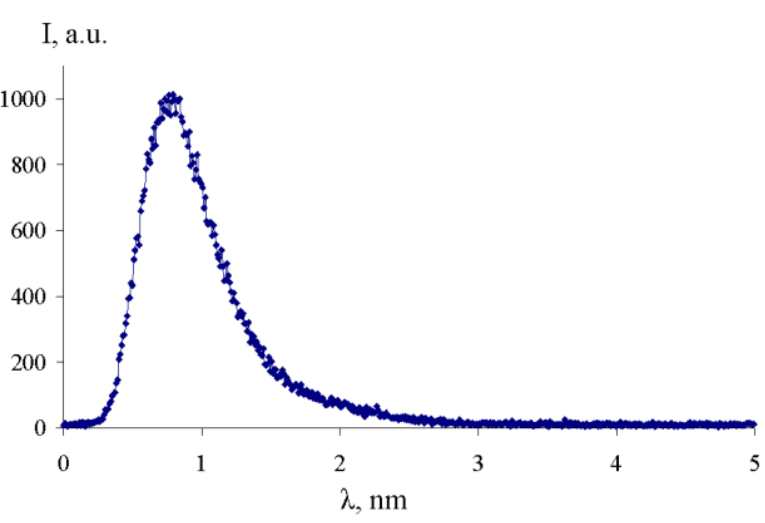

a

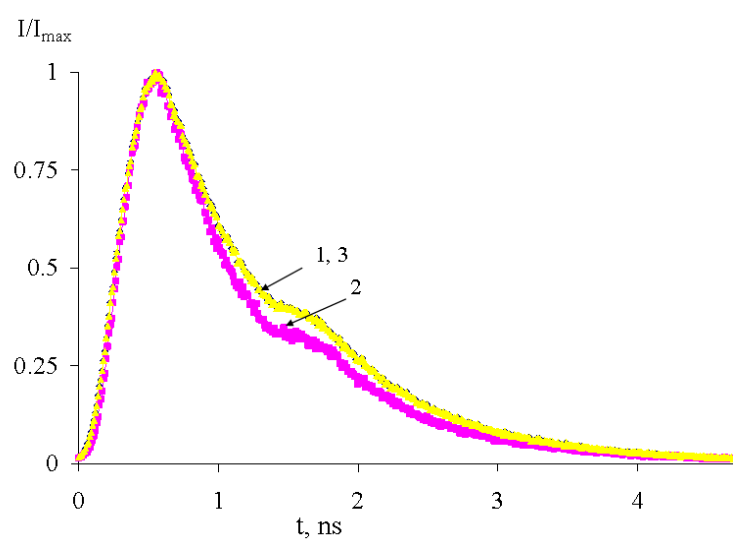

$\mathrm{b}$

Fig.5. Kinetics of fluorescence decay of dye 1 in solution (a) and in porous films of POA (b): 1 - samples without silver NPs; 2 - sample I; 3 - sample II.

For PAO films with a dye, the kinetic fluorescence curves have a certain inflection, which is observed at the decay of the luminescence intensity. The fluorescence lifetime of the dye in the films is $0.98 \mathrm{~ns}, 0.95 \mathrm{~ns}$, and $1.06 \mathrm{~ns}$ in the series of samples - without silver NPs, with NPs sample I and with NPs sample II, respectively (Fig. 5, b). In this case, for film II, an insignificant increase in the fluorescence lifetime is observed. An increase in the luminescence lifetime in the presence of silver NPs was also observed for an ethanol solution of 
merocyanine dye $\mathbf{1}$ and can be associated with the reverse energy transfer from NPs to the dye molecules [9]. The spectrum of stimulated emission of the dye in ethanol with $\mathrm{C}_{\mathrm{cr}}=2.5 \times 10^{-4}$ mol is shown in Fig. 6 , curve 1 . The length of the active medium in the quartz cell was $1 \mathrm{~cm}$. At a pump source power density (P) below 4 $\mathrm{MW} / \mathrm{cm}^{2}$, only spontaneous fluorescence of the dye with a half-width of the band $\Delta \lambda_{1 / 2}=30 \mathrm{~nm}$ is observed. At $\mathrm{P}=5.2 \mathrm{MW} / \mathrm{cm}^{2}$, a narrow lasing band with $\Delta \lambda_{1 / 2}=3 \mathrm{~nm}$ appears against the background of the fluorescence spectrum.

The lasing spectrum has three maxima at wavelengths of $677 \mathrm{~nm}, 679.5$ and $682 \mathrm{~nm}$. The observed maxima in the lasing spectrum are modes of laser radiation, since they are equidistant from each other [10]. The emission spectrum of the dye in PAO is shown in Fig. 6, curve 2. The stimulated emission spectrum has one maximum at $667 \mathrm{~nm}$. Dye radiation is generated at high values of the pump power density $\left(\sim 100 \mathrm{MW} / \mathrm{cm}^{2}\right)$. The reasons for such an ineffective dye generation may be cis-trans photoisomerization of merocyanine dye molecules [11], as well as the interaction of dye molecules with molecular oxygen [12].

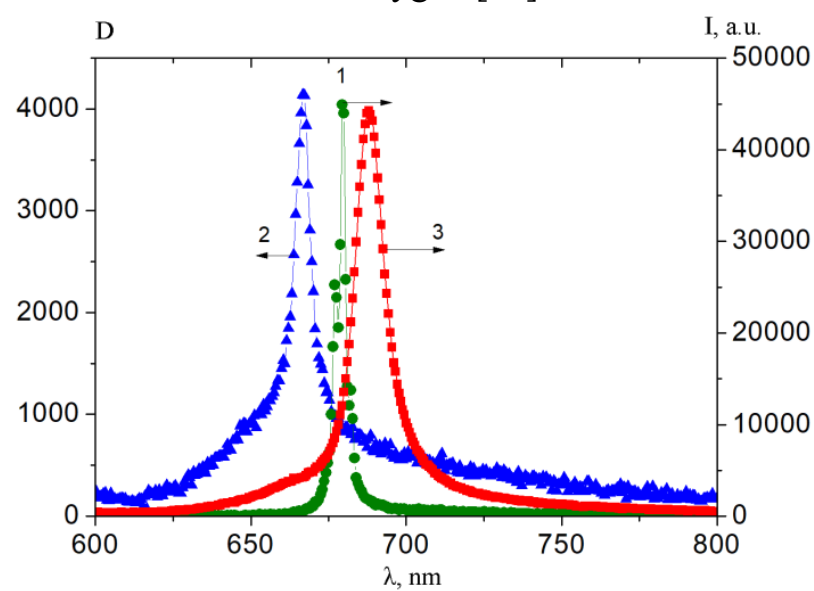

Fig.6. Spectra of stimulated emission of the dye in different media:

1 - in ethanol $\mathrm{C}=2.5 \cdot 10^{-4} \mathrm{~mol}, \mathrm{P}=5.2 \mathrm{MW} / \mathrm{cm}^{2} ; 2$ - in PAO matrix before surface modification, $\mathrm{P}=160 \mathrm{MW} / \mathrm{cm}^{2} ; 3$ - in PAO matrix with a PMMA film applied to the PAO surface, $\mathrm{P}=40 \mathrm{MW} / \mathrm{cm}^{2}$

Dependences of the FWHM (full width at half maximum) and the intensity of stimulated emission in the PAO on the value of $\mathrm{P}$ were plotted from the measured emission spectra (Fig.7). The values of generation threshold for the samples were determined. The lasing threshold was $103 \mathrm{MW} / \mathrm{cm}^{2}$ for PAO film with dye 1 without NPs (Fig. 7).

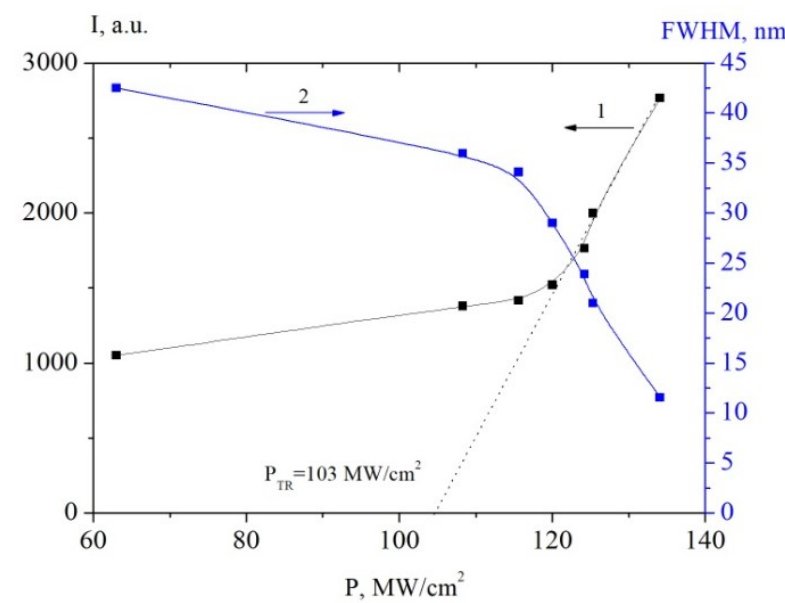

Fig.7. Dependence on the pump power density of the luminescence intensity (1) and the half-width of the emission band (2) of the dye in PAO $\left(\lambda_{\text {gen }}=532 \mathrm{~nm}\right)$.

The emission spectra of PAO films doped with dye $\mathbf{1}$ and Ag NPs were measured. The measurement results show the absence of stimulated emission in PAO films doped with Ag NPs. The generation of stimulated emission from the dye in PAO films doped with Au NPs was also not detected. PAO films and the technique of doping of films with gold NPs are described in the work [13]. It was proposed to deposit a polymer film of 
polymethyl methacrylate (PMMC) on the surface of the PAO for reducing number of dye molecules nonradiatively transforming into $S_{0}$ upon laser photoexcitation. It is the main polymer used for the manufacture of active laser media with dye molecules [14]. This was the reason for the choice the PMMC. The spectrum of stimulated emission in the modified film is shown in Fig.6, curve 3.

The addition of polymer leads to an increase in the stimulated emission intensity, a decrease in the lasing threshold to $3 \mathrm{MW} / \mathrm{cm} 2$, and a shift of the lasing maximum to longer wavelengths to $687 \mathrm{~nm}$. In this case, the value of Q-factor has similar values for films before the application of the polymer film and after its application $(\mathrm{Q} \approx 57)$. Measurements of the absorption, fluorescence, and fluorescence excitation spectra of the PAO films with dye 1 before and after the application of the PMMA polymer were taken. Fig. 8, a shows that the absorption band of the dye in the PAO matrix has a maximum at a wavelength of $\lambda=610 \mathrm{~nm}$.

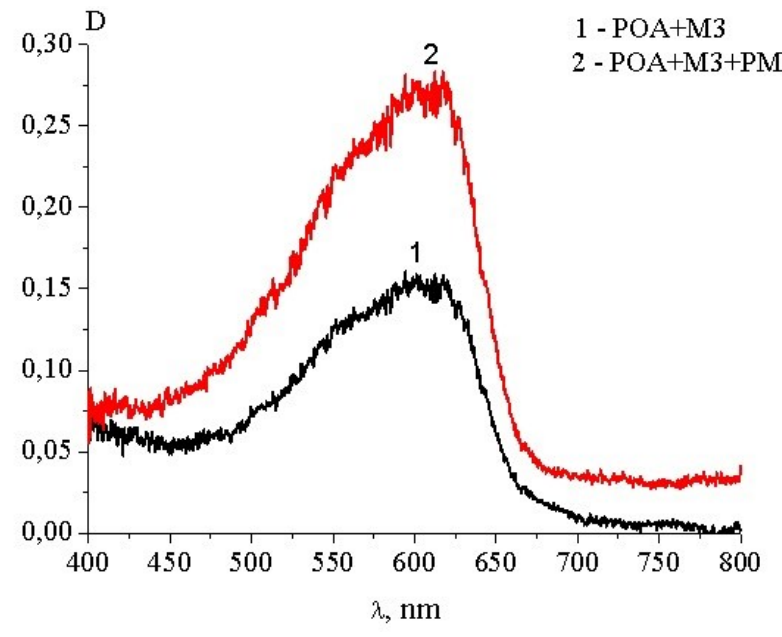

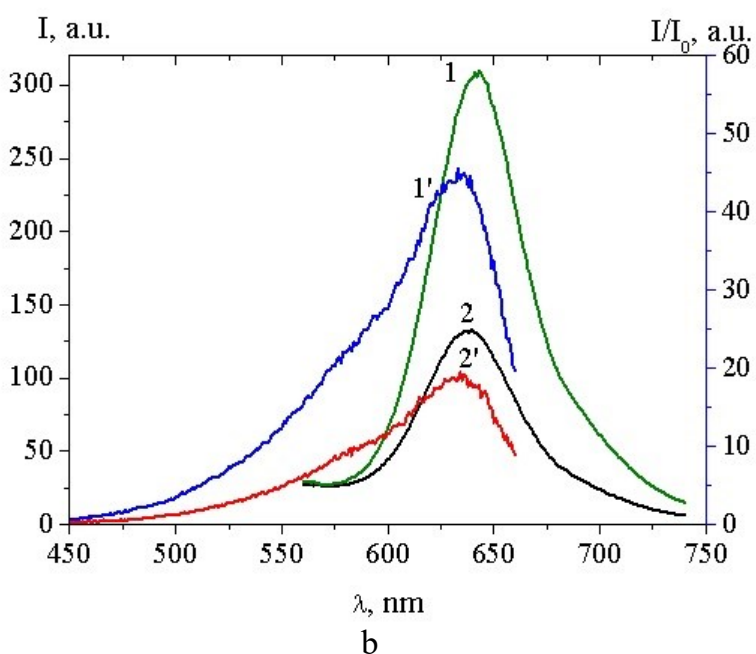

b

Fig.8. Absorption spectra $(1,2)(a)$, fluorescence $(1,2)$ and fluorescence excitation spectra $\left(1^{\prime}, 2^{\prime}\right)(b)$ of PAO films: 1, 1'$\mathrm{POA}+$ dye 1+PMMC Fl; 2, 2'-POA+ dye 1.

The deposition of the polymer on the surface of the PAO film does not change the shape of the absorption spectrum of the dye, but leads to a twofold increase in the optical density D. Since the concentration of dye molecules in the POA doesn't change. The increase in the value of D of the dye molecules can be associated with an increase in the extinction coefficient of the dye $\mathbf{1}$ when the PMMA polymer is applied to the surface of the POA. Photoexcitation of the fluorescence of the dye in the samples was carried out in the absorption band of the dye at a wavelength equal to $\lambda=640 \mathrm{~nm}$. The fluorescence spectrum and fluorescence excitation spectra show no changes in the shape of the spectra when the PMMC polymer is applied to the surface of the PAO. The intensity of the fluorescence spectra of the dye in the POA+PMMC films increases by a factor of 2.4 in comparison with the sample without PMMC. Thus, measurements of the absorption and fluorescence spectra of the dye in the PAO films showed an increase in the optical density in the absorption band of the dye and an additional increase in the fluorescence intensity of the dye in the PAO films with the polymer PMMC deposited on their surface.

\section{Conclusions}

The photonics of the merocyanine dye 1 in POA films was investigated. Low-Q stimulated emission of the dye is observed in the samples. The addition of silver nanoparticles to POA films leads to an increase in the absorption cross-section and an increase in the quantum yield of dye fluorescence in POA films. In the presence of silver and gold NPS in the pores of aluminum oxide, stimulated dye radiation wasn't observed. It is proposed to apply a PMMC polymer film to the surface of the POA for the reducing the influence of the external environment on the properties of the stimulated radiation of the dye in the POA films. There is a 2-fold increase in the optical density in the dye absorption band and a slight increase in the fluorescence intensity in samples with a PMMC film. The generation threshold of the merocyanine dye $\mathbf{1}$ is reduced by a factor of 40 in a POA sample with PMMC. But the $\mathrm{Q}$-factor of the resonator doesnt change $(\mathrm{Q} \approx 57)$. 


\section{Acknowledgements \\ This research is funded by the Science Committee of the Ministry of Education and Science \\ of the Republic of Kazakhstan (Grant No. AP08856161).}

\section{REFERENCES}

1 Diggle J.W., Downie T.C., Coulding C. W. Printing on Anodized Aluminium Surface. Chemical Reviews. 1969, Vol. 69, pp. $365-405$.

2 Shelkovnikov V.V., et al. Lasing of a solid-state active element based on anodized aluminum oxide film doped with rhodamine 6G. Russian Physics Journal. 2017, Vol. 59, No. 12, pp. 1989 - 1995.

3 Ibrayev N.Kh., Aimukhanov A.K. Influence of plasmon resonance in silver nanoparticles on the properties of stimulated emission of 1,3,5, 7,8 - pentamethyl - 2,6-diethylpyrromethene difluoroborate molecules in film of porous aluminum oxide. Optics and Laser Technology. 2019, Vol. 115, pp. $246-250$.

4 Afanasyev D.A., Ibrayev N.Kh., Omarova G.S., et al. Spectral-Luminescence and Lasing Properties of Merocyanine Dye Solutions in the Presence of Silver Nanoparticles. Optics and Spectroscopy. 2020, Vol. 128, No.1, pp.61 - 65.

5 Napolskii K.S., Barczuk P.J., Vassiliev S.Yu., et al. Templating of electrodeposited platinum group metals as a tool to control catalytic activity. Electrochimica Acta. 2007, Vol. 52, No. 28, pp. $7910-7919$.

6 Aimukhanov A.K., Ibrayev N. Kh. Influence of gold nanoparticles on the properties of stimulated emission of 6amino-1h-phenalen-1-one in the pores of anodized aluminum oxide. Journal of Luminescence. 2018, Vol. 204, pp.216 220 .

7 Kulinich A.V., Derevyanko N.A., Ishchenko A.A., et al. Synthesis and spectral properties of malononitrile-based merocyanine dyes. Russian Chemical Bulletin. 2019, Vol. 54, No. 12, pp. $2820-2830$

8 Bondarev S.L., et al. Fluorescence and solvatochromism of a merocyanine dye with a high quadratic polarizability in solutions and polymer films. Journal of Luminescence. 2007, Vol. 124, No. 1, pp.178 - 186.

9 Yang M., Moroz P., Jin Z., et al. Delayed photoluminescence in metal-conjugated fluorophores. JACS. 2019, Vol. 141, No. 28, pp.11286 - 11297.

10 Ibrayev N.Kh., Ishchenko A.A., Afanasyev D.A., Zhumabay N.D. Active laser medium for near-infrared spectral range based on electron-unsymmetrical polymethine dye and silver nanoparticles. Applied Physics B: Lasers and Optics. 2019, Vol. 125, pp. $1-7$.

11 Chibisov A.K., Zakharova G.V., Gorner H., et al. Photorelaxation Processes in Covalently Linked Indocarbocyanine and Thiacarbocyanine Dyes. Journal of Chemical Physics. 1995, Vol. 99, pp. $886-893$.

12 Butrimovich O.V., Voropay E.S., Ksenofontova N.M. Study of the photostability of active media based on polymethine dyes. All-Union Meeting: Inverse Population and Lasing on Transitions in Atoms and Molecules, 1986,52 p. [in Russian].

13 Omarova G.S., Afanasyev D.A., Ibrayev N. Kh. Generation of stimulated emission of electron-unsymmetrical polymethine dye in films of porous aluminum oxide doped with gold nanoparticles. Theoretical and Applied Science. 2019, Vol. 78, No. 10, pp. $351-358$.

14 Peterson O.G., Snavely B.B. Stimulated emission from flashlamp-exsited organic dyes in polymethyl methacrylate. Applied Physics Letters. 1968, Vol. 12, pp. 238 - 240. 